Pacific Journal of Mathematics

PATHS ON POLYHEDRA. I 


\section{PATHS ON POLYHEDRA. II}

\section{VICTOR KLEE}

Continuing the author's earlier investigation, this paper studies the behavior of paths on (convex) polyhedra relative to the facets of the polyhedra. In Section 1, the polytopes which are polar to the cyclic polytopes are shown to admit Hamiltonian circuits, and the fact that they do leads to sharp upper bounds for the lengths of simple paths or simple circuits on polyhedra of a given dimension having a given number of facets. Section 2 is devoted to the conjecture, due jointly to Philip Wolfe and the author, that any two vertices of a polytope can be joined by a path which never returns to a facet from which it has earlier departed. This implies a well-known conjecture of Warren Hirsch, asserting that $n-d$ is an upper bound for the diameter of $d$-dimensional polytopes having $n$ facets. The Wolfe-Klee conjecture is proved here for 3-dimensional polyhedra, and a stronger conjecture (dealing with polyhedral cell-complexes) is established for certain special cases.

Our notation and terminology are as in $[10,11,12,13] .^{1}$ In particular, a polyhedron is a set which is the intersection of finitely many closed halfspaces in a finite-dimensional real linear space, and a $d$-polyhedron is one which is $d$-dimensional. The faces of a polyhedron $P$ are the empty set, $P$ itself, and the intersections of $P$ with the various supporting hyperplanes of $P$. Two faces are incident provided one contains the other. The 0 -faces and 1 -faces of $P$ are its vertices and edges, and when $P$ is a $d$-polyhedron its $(d-1)$-faces and $(d-2)$ faces are called facets and subfacets respectively. A proper polyhedron is one which contains no line, or, equivalently (assuming it is not empty), one which has at least one vertex. A polytope is a bounded polyhedron; equivalently, it is a set which is the convex hull of a finite set of points. Two vertices of a polyhedron $P$ are adjacent provided they are joined by an edge of $P$. A path on $P$ is a finite sequence $\left(x_{0}, x_{1}, \cdots, x_{l}\right)$ of consecutively adjacent vertices, and the integer $l$ is the length of the path. The diameter of a polyhedron is the smallest number $l$ such that any two vertices of the polyhedron can be joined by a path of length $\leqq l .^{2}$

The present paper is part of a development of recent years in

Received November 19, 1964. Research supported in part by the National Science Foundation, U.S.A. (NSF-GP-3579).

1 Hirsch's conjecture is reported on p. 168 of [5]. See also p. 160 of [5] and pp. 608-610 of [10]. And see footnote 16 at end of paper.

2 Diameters of polyhedra are studied in $[\mathbf{1 0}, \mathbf{2 5}]$. 
which the importance of linear programming has reawakened interest in the area forming the geometric background of the subject, namely the study of the facial structure of polyhedra. ${ }^{3}$ Because of the special importance of the simplex method, the study of paths on polyhedra is especially relevant. Several authors have considered the behavior of such paths relative to the number of vertices of the polyhedron $[2,9,10,12,16]$. However, in a practical linear programming problem one has much more direct information about the number of facets of the feasible region than about the number of its vertices, so it seems appropriate to study the behavior of paths relative to the number of facets. Such a study was initiated in $[10,12\rfloor$ and is continued here.

1. Longest simple paths and circuits. A path $\left(x_{1}, x_{1}, \cdots, x_{l}\right)$ is called a simple path provided no vertex is repeated; it is a simple circuit provided $l \geqq 2$ and $x_{l}=x_{0}$ but there is no other repetition among the $x_{i}$ 's. A Hamiltonian path or Hamiltonian circuit on a polyhedron $P$ is a simple path or circuit which runs through all the vertices of $P_{0}{ }^{1}$

Two $d$-polyhedra $P$ and $Q$ are said to be combinatorially equivalent provided there is a biunique correspondence between the faces of $P$ and the faces of $Q$ such that both incidence and dimension are preserved. Two polytopes $P$ and $Q$ will be called combinatorially dual provided there is a bianique correspondence between their faces such that incidence is preserved and dimension is complemented, so that the $s$-faces of one polytope correspond to the $(d-1-s)$-faces of the other.

The moment curve $M_{d}$ is the subset of $\boldsymbol{R}^{d}$ consisting of all points of the form $\left(r, r^{2}, \cdots, r^{d}\right)$ for $r \in \boldsymbol{R}^{5}$ A cyclic d-polylope is one which is combinatorially equivalent to a $d$-polytope whose vertices are all on $M_{a}[6]$.

THEOREM 1.1. If a d-polylope is a cyclic d-polytope or is combinatorially dual to a cyclic d-polylope, then it admits a Hamillonian circuit.

Proof. Since the 2-dimensional case is trivial, we assume that $d \geqq 3$. Consider a set $V$ of at least $d+1$ points on $M_{d}$, and let $P \cdot=$ con $V .^{6}$ It is known that each point of $V$ is a vertex of $P$, and

${ }^{3}$ For a survey of some of the interconnections, see [13].

4 For results on the existence or nonexistence of Hamiltonian paths ur circuits in 3-polytopes, see $[\mathbf{2}, \mathbf{9}, \mathbf{2 0}, \mathbf{2 1}, \mathbf{2 2}, \mathbf{2 3}]$.

${ }_{5} \boldsymbol{R}$ denotes the real number field.

${ }^{6}$ Equality by definition is indicated by $\cdot=$ or $=\cdot$. 
that for $d \geqq 4$ each two vertices of $P$ are joined by an edge of $P$ $[4,6,17]$, whence of course $P$ admits a Hamiltonian circuit. It is known [6] that the facets of $P$ are all simplices, each having $d$ vertices, and that a set $F$ of $d$ points of $V$ determines a facet of $P$ if and only if between each two points of $V \sim F$ there is (in the natural ordering on $M_{d}$ ) an even number of points of $F .^{7}$ From this it is easy to identify the edges of $P$ when $d=3$, and to see that $P$ admits a Hamiltonian circuit. That disposes of the cyclic polytopes.

Now we want to produce a Hamiltonian circuit on a polytope $Q$ which is combinatorially dual to a cyclic polytope $P$, but rather than working directly with the vertices and edges of $Q$ we may consider the facets and subfacets of $P$. It suffices to show that if $P$ is as in the preceding paragraph, then all of the facets of $P$ can be arranged in a sequence $\left(F_{0}, \cdots, F_{l}\right)$ such that $F_{l}=F_{0}$, there is no other repetition among the $F_{i}^{\prime}$ 's, and $F_{i-1} \cap F_{i}$ is a subfacet for $1 \leqq i \leqq l$. Since all the facets of $P$ are simplices, the last condition is equivalent to the requirement that there are $d-1$ vertices common to $F_{i-1}$ and $F_{i}$. Recalling Gale's characterization [6] of the facets of $P$, we see that the problem at hand is purely combinatorial in nature.

For $2 \leqq d<n$, let $V_{n} \cdot=\{1, \cdots, n\}$ and let $\boldsymbol{F}(d, n)$ denote the class of all $d$-pointed sets $F$ in $V_{n}$ such that between each two points of $V_{n} \sim F$ there is an even number of points of $F$. The initial parity of $F$ is the parity of the set of all points of $F$ which precede the first point of $V_{n} \sim F$. The set of all members of $\boldsymbol{F}(d, n)$ which have odd [resp. even] initial parity will be denoted by $\boldsymbol{F}_{0}(d, n)\left[\operatorname{resp} . \boldsymbol{F}_{e}(d, n)\right]$. An ordered pair $(F, G)$ of members of $\boldsymbol{F}_{0}(d, n)$ will be called admissible provided $F \cap G$ consists of exactly $d-1$ points.

Lemma. Suppose $2 \leqq d<n$. Let $A$ consist of the first $d$ points of $V_{n}$. If $d$ is even let $Z$ consist of the last $d$ points of $V_{n}$, and if $d$ is odd let $Z$ consist of the first point and the last $d-1$ points of $V_{n}$. Then the members of $\boldsymbol{F}(d, n)$ which have the same initial parity as $A$ and $Z$ can be arranged without repetition in a sequence $\left(F_{0}, \cdots, F_{k}\right)$ such that $F_{0}=A, F_{k}=Z$, and $\left(F_{i-1}, F_{i}\right)$ is admissible for $1<i \leqq k$.

Proof of the lemma. Let the assertion of the lemma be denoted by $L(d, n)$. For $d=2$, the assertion is obvious; we merely take $F_{i} \cdot=\{i+1, i+2\} \subset V_{n}$. For $d=3$, we keep the point 1 fixed and apply the preceding pattern to the remaining two points; that is, $F_{i} \cdot=$

7 Gale's proof [6] is given only for the even-dimensional case, but it can be extended without difficulty to the general case. 
$\{1, i+2, i+3\}$. The same procedure will carry us from any even value of $d$ to the next odd value, and it remains only to go from an even value to the next even value. Specifically, we suppose that $L(d, n)$ is known for a given even $d$ and for all $n>d$, and we want to establish $L(d+2, m)$ for an arbitrary $m>d+2$. This is accomplished by successive applications of

$$
L(d, m-2), L(d, m-3), \cdots, L(d, d+1),
$$

running first through the members of $\boldsymbol{F}(d+2, m)$ which contain $\{1,2\}$, next through those which contain $\{2,3\}$ but not $\{1\}$, etc. The procedure is illustrated below for the case in which $d+2=6$ and $m=$ 10; the reader can easily supply the formal details for the general argument.

$$
\begin{aligned}
& L(4,8)\left(\begin{array}{ccccccccccc}
1 & 2 & 3 & 4 & 5 & 6 & 7 & 8 & 9 & 10 & \\
x & x & x & x & x & x & 0 & 0 & 0 & 0 & A \\
x & x & 0 & 0 & 0 & 0 & x & x & x & x &
\end{array}\right. \\
& L(4,7)\left(\begin{array}{llllllllll}
0 & x & x & 0 & 0 & 0 & x & x & x & x \\
0 & x & x & x & x & x & x & 0 & 0 & 0
\end{array}\right. \\
& L(4,6)\left(\begin{array}{llllllllll}
0 & 0 & x & x & x & x & x & x & 0 & 0 \\
0 & 0 & x & x & 0 & 0 & x & x & x & x
\end{array}\right. \\
& L(4,5)\left(\begin{array}{lllllllllll}
0 & 0 & 0 & x & x & 0 & x & x & x & x & \\
0 & 0 & 0 & x & x & x & x & x & x & 0 & \\
0 & 0 & 0 & 0 & x & x & x & x & x & x & Z
\end{array}\right.
\end{aligned}
$$

To complete the proof of 1.1, we show that all of the members of $\boldsymbol{F}(d, n)$ can be arranged in a sequence $\left(F_{0}, \cdots, F_{l}\right)$ such that $F_{l}=$ $F_{0}$, there is no other repetition among the $F_{i}$ 's, and the pair $\left(F_{i-1}, F_{i}\right)$ is admissible for $1 \leqq i \leqq l$. Let

$$
\begin{gathered}
A \cdot=\{i: 1 \leqq i \leqq d\}, \quad B \cdot=(i: n-d+1 \leqq i \leqq n\}, \\
C \cdot=\{i: 1 \leqq i \leqq d) \cup\{n\}, \text { and } D \cdot=\{1\} \cup\{i: n-d+1<i \leqq n\},
\end{gathered}
$$

all members of $\boldsymbol{F}(d, n)$. Then the sequence $\left(F_{0}, \cdots, F_{l}\right)$ is formed as follows, using the patterns described in the lemma:

When $d$ is odd, run through $\boldsymbol{F}_{0}(d, n)$ from $A$ to $D$; next go directly to $B$; then run through $\boldsymbol{F}_{e}(d, n)$ from $B$ to $C$, and finally return to $A$; When $d$ is even, run through $\boldsymbol{F}_{e}(d, n)$ from $A$ to $B$; next go directly to $D$; then run through $\boldsymbol{F}_{0}(d, n)$ from $D$ to $C$, and finally return to $A$. The procedure is illustrated below with $n=14$ and $d=5$ or 6 . 


\begin{tabular}{|c|c|c|c|c|c|c|c|c|c|c|c|c|c|c|c|}
\hline \multirow{3}{*}{$L(5,14)$} & 1 & 2 & 3 & 4 & 5 & 6 & 7 & & & 10 & 11 & 12 & 13 & 14 & \\
\hline & $x$ & $x$ & $x$ & $x$ & $x$ & 0 & 0 & 0 & 0 & 0 & 0 & 0 & 0 & 0 & $A$ \\
\hline & $x$ & 0 & 0 & 0 & 0 & 0 & 0 & 0 & 0 & 0 & $x$ & $x$ & $x$ & $x$ & $D$ \\
\hline \multirow{3}{*}{$L(5,14)$} & 0 & 0 & 0 & 0 & 0 & 0 & 0 & 0 & 0 & $x$ & $x$ & $x$ & $x$ & $x$ & $B$ \\
\hline & $x$ & $x$ & $x$ & $x$ & 0 & 0 & 0 & 0 & 0 & 0 & 0 & 0 & 0 & $x$ & $C$ \\
\hline & $x$ & $x$ & $x$ & $x$ & $x$ & 0 & 0 & 0 & 0 & 0 & 0 & 0 & 0 & 0 & $A$ \\
\hline \multirow{2}{*}{$L(6,14)$} & $x$ & $x$ & $x$ & $x$ & $x$ & $x$ & 0 & 0 & 0 & 0 & 0 & 0 & 0 & 0 & $A$ \\
\hline & 0 & 0 & 0 & 0 & 0 & 0 & 0 & 0 & $x$ & $x$ & $x$ & $x$ & $x$ & $x$ & $B$ \\
\hline \multirow{3}{*}{$\mathrm{L}(5,13)$} & $x$ & 0 & 0 & 0 & 0 & 0 & 0 & 0 & 0 & $x$ & $x$ & $x$ & $x$ & $x$ & $D$ \\
\hline & $x$ & $x$ & $x$ & $x$ & $x$ & 0 & 0 & 0 & 0 & 0 & 0 & 0 & 0 & 0 & $C$ \\
\hline & $x$ & $x$ & $x$ & $x$ & $x$ & $x$ & 0 & 0 & 0 & 0 & 0 & 0 & 0 & 0 & $A$ \\
\hline
\end{tabular}

Again, the formal description is left to the reader. ${ }^{8}$

Now (following the pattern of [10] and [12]) let us denote the class of all $d$-polytopes by $\boldsymbol{P}_{d}$, while the subclasses $\boldsymbol{P}_{d}^{v}$ and $\boldsymbol{P}_{d}^{f}$ consist respectively of the $d$-polytopes which are simple (each vertex incident to $d$ edges) and those which are simplicial (each facet a simplex). For each polyhedron $P$, let $\lambda(P)$ [resp. $\kappa(P)$ ] denote the largest number which is realized as the length of a simple path [resp. simple circuit] on $P$. We are interested in the maxima of $\lambda(P)$ and $\kappa(P)$ as $P$ ranges over various subclasses of $\boldsymbol{P}_{d}$. Let us define

$$
\begin{aligned}
& \Lambda_{r}(d, n) \cdot=\max \left\{\lambda(P): P \in \boldsymbol{P}_{a} \text { and } f_{r}(P) \leqq n\right\} \text { and } \\
& K_{r}(d, n) \cdot=\max \left\{\kappa(P): P \in \boldsymbol{P}_{d} \text { and } f_{r}(P) \leqq n\right\},
\end{aligned}
$$

where $f_{r}(P)$ denotes the number of $r$-faces of $P$. Similarly, we define $\Lambda_{r}^{v}$ and $K_{r}^{v}$ (where $P_{d}$ is replaced by $\boldsymbol{P}_{d}^{v}$ ) as well as $\Lambda_{r}^{f}$ and $K_{r}^{f}$ (where $\boldsymbol{P}_{d}$ is replaced by $\left.\boldsymbol{P}_{d}^{f}\right)$. Our attention will be confined to two possible values for $r$-namely, $r=0$ and $r=d-1$-and the results are as follows: ${ }^{9}$

$$
\begin{array}{ll}
1.2 & K_{0}(d, n)=K_{0}^{f}(d, n)=n=\Lambda_{0}^{f}(d, n)+1=\Lambda_{0}(d, n)+1 . \\
1.3 \quad(d-1)\left[\frac{n-2}{d-1}\right]+2 \leqq K_{0}^{v}(d, n) \leqq \Lambda_{0}^{v}(d, n)+1 \leqq n
\end{array}
$$

with equality throughout if $d \leqq 3$ or $n \equiv 2 \bmod (d-1)$.

${ }^{8}$ It would be interesting to know which of the $m$-neighborly $(2 m)$-polytopes studied by Gale [6] and Grünbaum [8] are such that their combinatorial duals admit Hamiltonian paths or circuits.

9 These results are stated only for polytopes, but they can easily be reformulated so as to apply to polyhedra; 4.5 of [11] is useful in this connection. 
$1.4 \quad K_{d-1}(d, n) \geqq K_{d-1}^{n}(d, n) \geqq\left(\begin{array}{l}n-\left[\frac{d+1}{2}\right] \\ n-d\end{array}\right)+\left(\begin{array}{l}n-\left[\frac{d+2}{2}\right] \\ n-d\end{array}\right) \leqq$

$$
\leqq \Lambda_{d-1}^{v}(d, n)+1 \leqq \Lambda_{d-1}(d, n)+1 \text {, }
$$

with equality throughout if $d \leqq 8$, or $n \leqq d+3$, or $n \geqq(d / 2)^{2}-1$, or the MJSG conjecture is true.

$$
\Lambda_{d-1}^{f}(d, n)+1 \geqq K_{d-1}^{f}(d, n) \geqq\left[\frac{n-2}{d-1}\right]+d,
$$

with equality throughout if $d \leqq 3$ or $n \leqq d+3$.

These estimates for $K$ and $\Lambda$ may be compared with estimates for the diameter function $\Delta$ given in $|10,25|$ and for the height funetions $H, S$ and $Z$ given [12].

Proofs of 1.2-1.5. The truth of 1.2 follows immediately from the fact that the cyclic polytopes admit Hamiltonian circuits.

For 1.3 we define $g(n) \cdot=(d-1)(n-d)+2$ and consider the $d$ polytopes $Q_{n}$ formed as follows: $Q_{d+1}$ is a $d$-simplex with Hamiltonian circuit $\left(x_{1}, x_{2}, \cdots, x_{g(a+1)}, x_{1}\right)$; given a simple $d$-polytope $Q_{n}$ with Hamiltonian circuit $\left(x_{1}^{n}, x_{2}^{n}, \cdots, x_{g(n)}^{n}, x_{1}^{n}\right)$, we form $Q_{n+1}$ by truncating $Q_{n}$ at the vertex $x_{g(n)}$, and thus replacing $x_{g(n)}$ by a new facet which is a $(d-1)$-simplex whose vertices lie on the $d$ edges of $Q_{n}$ which issue from $x_{g^{\prime}(n)}^{n}$. The $d$-polytope $Q_{n+1}$ is simple, has $g(n+1)$ vertices, and admits a Hamiltonian circuit

$$
\left(x_{1}^{n}, x_{2}^{n}, \cdots, x_{g(n)-1}^{n}, x_{y(n)}^{n+1}, x_{y(n)+1}^{n+1}, \cdots, x_{g(n+1)}^{n+1}, x_{1}^{n}\right),
$$

where $x_{y\langle n\rangle}^{n+1} \in\left|x_{g(n)-1}^{n}, x_{y(n)}^{n}\right|$ and $\left.x_{y\langle n+1)}^{n+1} \in\right] x_{g / n)}^{n}, x_{1}^{n}[$. (With additional care, we can also insure that $Q_{n}$ admits a Hamiltonian path which is a " maximum gradient" path.) For the details of this construction and for the rest of the proof of 1.3, see the proof of (3) in [12].

To establish all of the inequalities of 1.4 , it suffices to show that $K_{d-1}^{v}(d, n) \geqq \mathscr{P}(d, n)$, where

$$
\rho(d, n) \cdot=\left(\begin{array}{l}
n-\left[\frac{d+1}{2}\right] \\
n-d
\end{array}\right)+\left(\begin{array}{l}
n-\left[\frac{d+2}{2}\right] \\
n-d
\end{array}\right) .
$$

Gale [6] has proved that a cyclic $d$-polytope with $n$ vertices is simplicial and has exactly $\rho(d, n)$ facets ${ }^{7}$, whence the dual polytopes are simple and have $n$ facets and $\rho(d, n)$ vertices. Since the dual polytopes admit Hamiltonian circuits by 1.1 , the desired inequality follows. (This shows also that equality holds in 1.3 whenever $n=\varphi(d, m)$ for some $m>d$.) For equality in 1.4, it suffices to show that if a 
$d$-polytope has at most $n$ facets, then it has at most $\varphi(d, n)$ vertices. This statement was called the "JSG conjecture" in [11], but should have been called the $M J S G$ conjecture because of its first appearance in [17]. It has been established for $n \leqq d+3$ [7] and $n \geqq(d / 2)^{2}-1$ [11], hence in particular for $d \leqq 6$. And see [8] for $d=7,8$.

For 1.5 it suffices to follow the proof of (7) in [12], noting that Hamiltonian circuits are admitted by the polytopes $P_{n}$ which are constructed there. (They also admit " maximum gradient" Hamiltonian paths.) Alternatively, we could employ simplicial $d$-polytopes $Q_{n}^{\prime}$ which are dual to the simple polytopes $Q_{n} ; Q_{d+1}^{\prime}$ is a $d$-simplex and $Q_{n+1}^{\prime}$ is formed by adding a pyramidal cap over one of the facets of $Q_{n}^{\prime}$.

For equality in 1.5 when $d>3$, it would suffice to show that if a simplicial $d$-polytope has $n$ vertices, then it has at least $(d-1)(n-d)+2$ facets. The equivalent dual statement was made by Brückner [3] for the 4-dimensional case, but his proof was incorrect (as noted in [18]). Grünbaum [8] has settled the case $n \leqq d+3$.

2. The existence of $W_{v}$ paths. Consider a path $\left(x_{0}, x_{1}, \cdots, x_{l}\right)$ on a polyhedron $P$, and for $1 \leqq i \leqq l$ let $\sigma_{i}$ denote the edge $\left[x_{i-1}, x_{i}\right]$. The path will be called a $W_{v}$ path or a $W_{e}$ path provided the following respective conditions are satisfied:

$\left(W_{v}\right)$ if $i<j<k$ and a facet $F$ of $P$ includes both $x_{i}$ and $x_{k}$, then $F$ includes $x_{j}$ also;

$\left(W_{e}\right) \quad \sigma_{i-1} \neq \sigma_{i}(1 \leqq i \leqq l)$; if $i<j<k$ and a facet $F$ of $P$ contains both $\sigma_{i}$ and $\sigma_{k}$, then $F$ contains $\sigma_{j}$ also.

Every $W_{v}$ path is simple. A $W_{e}$ path of length $l$ need not be simple, but it must involve $l$ distinct edges. These and some related types of paths are considered in [14]. Here we are interested mainly in $W_{v}$ paths, for the conjecture formulated in the introduction may be stated more formally as follows: Any two vertices of a polytope can be joined by a $W_{v}$ path. This will be called the $W_{v}$ conjecture. The connection between the $W_{v}$ conjecture and Hirsch's conjecture ${ }^{1}$ arises from the following remark.

2.1. If $P$ is a d-polyhedron which has at most $n$ facets and $l$ is the length of a $W_{v}$ path [resp. $W_{e}$ path] on $P$, then $l \leqq n-d$ $[$ resp. $l \leqq n-d+2]$.

Proof. There are facets $G_{i}$ such that

$$
x_{0} \notin G_{0} x_{l} \in G_{0} \sim G_{1}, \cdots, x_{l-1} \in G_{l-2} \sim G_{l-1}, x_{l} \in G_{l-1} .
$$

From condition $\left(W_{v}\right)$ it follows that the $G_{i}$ 's are distinct and $x_{0}$ is not included in any of the facets $G_{0}, \cdots, G_{l-1}$. Since there are at least $d$ facets of $P$ which include $x_{0}$, we have $n \geqq d+l$. A similar argument 


\section{[14] applies to $W_{e}$ paths.}

For $n>d$, there exist simple $d$-polytopes which have exactly $n$ facets and which admit $W_{v}$ paths of length $n-d$. For example, the $d$-polytopes $Q_{n}$ of $\S 1$ have this property. Note also that Hirsch's estimate for the diameter of a polyhedron cannot be improved in general, since for $n>d$ there exists a simple $\vec{d}$-polyhedron $J_{n}$ which has $n$ facets and is of diameter $n-d$. To start the construction, let $J_{d+1}$ be a half-cylinder over a $(d-1)$-simplex; that is, $J_{d+1}$ is the linear sum in $\boldsymbol{R}^{d}$ of a $(d-1)$-simplex and a ray which is not parallel to the hyperplane determined by the simplex. Now suppose that we have constructed a simple $d$-polyhedron $J_{n}$ such that any two vertices of $J_{n}$ can be joined by a path of length $\leqq n-d$ and there are two vertices $x_{n}$ and $z_{n}$ of $J_{n}$ which cannot be joined by any shorter path. Suppose further that $x_{n}$ is incident to an unbounded edge $\rho_{n}$ of $J_{n}$ and to $d-1$ bounded edges whose other endpoints are $y_{n}^{1}, \cdots, y_{n}^{d-1}$. Then $J_{n+1}$ is formed by truncating $J_{n}$ at the vertex $z_{n}$, thus replacing $z_{n}$ by a facet which is a $(d-1)$-simplex having vertices $z_{n+1} \in \rho_{n} \sim\left\{z_{n}\right\}$ and $\left.y_{n+1}^{i} \in\right] y_{n}^{i}, z_{n}\left[\right.$. Let $x_{n+1} \cdot=x_{n}$. Since the only approach to $z_{n+1}$ along the edges of $J_{n+1}$ is through the vertices $y_{n+1}^{i}$, it is evident that $x_{n+1}$ and $z_{n+1}$ cannot be joined in $J_{n+1}$ by a path of length $<n+1-d$. Thus the induction can be carried through and the $d$-polyhedra $J_{n}$ can be constructed as described. ${ }^{10}$

In discussing the $W_{v}$ conjecture, we employ the notion of a polyhedral cell-complex (or simply complex), where this is a finite family $\boldsymbol{K}$ of polyhedra (the cells of $\boldsymbol{K}$ ) in a finite-dimensional real linear space such that each face of a cell of $\boldsymbol{K}$ is itself a cell of $\boldsymbol{K}$, and the intersection of any two cells of $\boldsymbol{K}$ is a face common to both. ${ }^{11}$ If $P$ is a polyhedron, the family of all faces of $P$ forms a complex, as does the family of all faces other than $P$ itself; the latter complex will be demoted by $\boldsymbol{B}(P)$ and will be called the boundary complex of $P$. The notions of vertex, edge and path are defined for complexes in the obvious way, and a path $\left(x_{0}, x_{1}, \cdots, x_{l}\right)$ in a complex will be called a $W_{v}$ path provided the following condition is satisfied: if $i<j<k$ and a cell of $\boldsymbol{K}$ includes both $x_{i}$ and $x_{k}$, then it includes $x_{j}$ also. The $W_{e}$ paths in $\boldsymbol{K}$ are similarly defined. These requirements may appear to be stronger than those for $W_{v}$ paths in polyhedra, since they are not restricted to cells of a particular dimension. However, we note the following fact.

${ }^{10}$ At least for $d \leqq 3$, the unboundedness of the polyhedra in this construction is essential, for when $d \leqq 3$ the maximum diameter of $d$-polytopes having $n$ facets is $[n(d-1) / d]-d+2[\mathbf{1 0}]$.

${ }^{11}$ This is simply a finite geometric cell-complex in the sense of Alexandroff \& Hopf [1], except that the cells are not here required to be bounded. 
2.2. If $P$ is a cell of a polyhedral cell-complex $\boldsymbol{K}$, then every $W_{v}$ path on $P$ is a $W_{v}$ path in $K$. The same is true of $W_{e}$ paths.

Proof. Since the two situations are similar, we consider only the former. Let $\left(x_{0}, \cdots, x_{l}\right)$ be a $W_{v}$ path in $P$, and suppose a cell $C$ of $K$ includes $x_{i}$ and $x_{k}$ but not $x_{j}$, where $i<j<k$. Then the same is true of $C \cap P$, which is a proper face of $P$. Since $C \cap P$ is the intersection of all the facets of $P$ which contain $C \cap P$, there must be a facet of $C \cap P$ which includes $x_{i}$ and $x_{k}$ but not $x_{j}$. This is a contradiction and completes the proof.

The following useful fact was noted by Clyde Kendall and Johns Rulifson.

Proposition (Kendall and Rulifson) 2.3. Suppose that $\boldsymbol{K}$ is a polyhedral cell-complex in the Euclidean plane, and that the vertex $x$ of $\boldsymbol{K}$ can be joined to the vertex $y$ by a path in $\boldsymbol{K}$. Among all paths from $x$ to $y$ in $\boldsymbol{K}$, let $I$ be one of minimum Euclidean length. Then $\Pi$ is a $W_{v}$ path.

Proof. The result is an immediate consequence of the fact that if $C$ is an open convex set in the Euclidean plane and $u$ and $v$ are boundary points of $C$ which can be connected by an arc in the complement of $C$, then the shortest such connecting arc lies in the boundary of $C$.

With the aid of 2.3, we can almost prove the $W_{v}$ conjecture for 3-polyhedra.

THEOREM 2.4. Suppose that $x$ and $y$ are vertices of a 3-polyhedron $P$. If $P$ is unbounded, then $x$ and $y$ can be joined by a $W_{v}$ path on $P$. If $P$ is bounded and $F$ is a facet of $P$, then $x$ and $y$ can be joined by a path on $P$ for which no facet other than $F$ violates the $W_{v}$ condition..$^{12}$ (See footnote 17 at end of paper.)

Proof. If $P$ is bounded, we merely apply 2.3 to the Schlegel diagram of $P$ in $F$. This is obtained by choosing a point $z$ outside $P$ but near an inner point of $F$, so that for each point $w$ of $P \sim F$ the intersection $[w, z] \cap F$ consists of an inner point $\psi(w)$ of $F$. The projection $\psi$ takes the complex $\{C: C \in \boldsymbol{B}(P) \sim\{F\}\}$ onto a complex $\boldsymbol{K}$ in the plane of $F$, and then the desired conclusion follows from 2.3.

Now suppose that $P$ is unbounded and assume without loss of

${ }^{12}$ That is, $x$ and $y$ are joined by a path $\left(x_{0}, x_{1}, \cdots, x_{l}\right)$ such that if $i<j<k$ and $G$ is a facet of $P$ which includes $x_{i}$ and $x_{k}$, then $G$ includes $x_{j}$ or $G=F$. 
generality that $P$ lies in a 3 -dimensional real linear space $E . A s$ is easily verified, the interior of $P$ contains a ray $\rho$ which issues from a boundary point of $P$. We may assume that the boundary point is the origin 0 , whence $\rho=] 0, \infty[u$ for some $u \in \operatorname{int} P$. Let $H$ be a plane which supports $P$ at 0 , whence $E=H+\mathbb{R} u$ and each point $q$ of $E$ admits a unique expression in the form $q=q_{h}+q_{r} u$ with $q_{h} \in H$ and $q_{r} \in \boldsymbol{R}$. Let $V$ denote the (finite) set of all vertices of $P$ and let $m=\max \left\{v_{r}: v \in V\right\}+1$, whence $V$ is contained in the half-open strip $H+[0, m[u$ and thus the same is true of every bounded face of $P$. For each point $q \in H+[0, m[u$, let $\psi(q)$ denote the point at which $H$ is intersected by the ray from $m u$ through $q$. For each proper face $C$ of $P$, let $C_{H} \cdot=\psi(C \cap(H+[0, m[u)$. It can be verified that $\left\{C_{H}: C \in \boldsymbol{B}(P)\right\}$ is a cell-complex which is combinatorially equivalent to the boundary complex $\boldsymbol{B}(P)$, and hence the desired conclusion follows from 2.3.

In order to complete the proof of the $W_{v}$ conjecture for 3-polyhedra, we still must settle (rather than merely "almost settle") the case of 3-polytopes.

THEOREM 2.5. Any two vertices of a 3-polytope $P$ can be joined by a $W_{v}$ path on $P$. (See footnote 17 at end of paper.)

Proof. The proof is by induction on the number $n$ of facets of $P$, the assertion being obvious when $n=4$ for then $P$ is a tetrahedron. Suppose that $n>4$ and that the theorem has been proved for all 3polytopes having fewer than $n$ facets. Consider an arbitrary 3-polytope $Q$ which has $n$ facets. By a theorem of Steinitz \& Rademacher [19] ${ }^{13}$, the complex $\boldsymbol{B}(Q)$ is (combinatorially equivalent to one which is) obtained by means of a facet-splitting of type 1, 2 or 3 from a complex

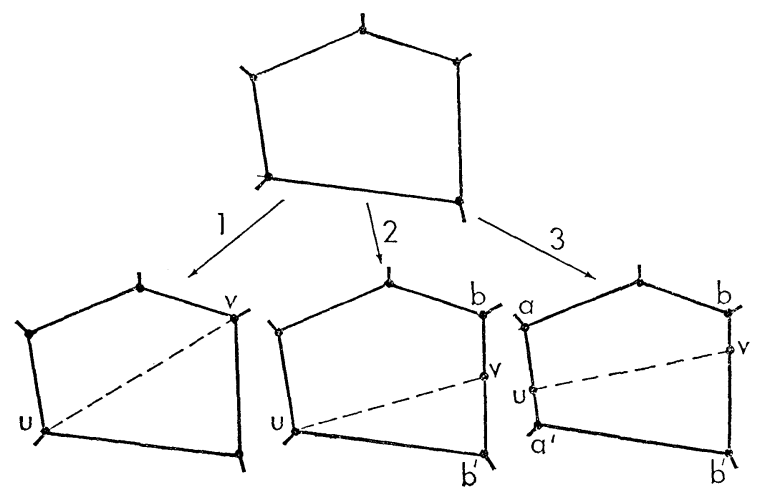

Figure 1

${ }^{13}$ See also Lyusternik [15]. 
$\boldsymbol{B}(P)$, where $P$ is a 3 -polytope having $n-1$ facets. The three types of facet-splitting are depicted in Figure 1 above. Each involves the addition of a new edge $[u, v]$, cutting across one of the facets of $P$; a facet-splitting of type $i$ involves also the addition of $i-1$ new vertices and the consequent splitting of $i-1$ of the edges of $P$. We shall follow the notation of Figure 1.

Now consider an arbitrary pair $x$ and $y$ of vertices of $Q$; we want to show that they can be joined by a $W_{v}$ path $\Pi$ on $Q$. If $\{x, y\}=$ $\{u, v\}$, we let $\Pi=(x, y)$. Now suppose that $x$ and $y$ are both vertices of $P$, whence (according to the inductive hypothesis) they are joined by a $W_{v}$ path $\Pi_{1}$ on $P$. If every edge of $\Pi_{1}$ is also an edge of $Q$, let $\Pi_{2}=\Pi_{1}$. Alternatively, some edge(s) of $\Pi_{1}$ may be split in the transition from $P$ to $Q$, and in this case $\Pi_{2}$ is obtained from $\Pi_{1}$ by the corresponding replacement(s) of one edge of $P$ by two edges of $Q$. (For example, $\cdots, b, v, b^{\prime}, \cdots$ appears in $\Pi_{2}$ if $\cdots, b, b^{\prime}, \cdots$ appears in $\Pi_{1}$.) Then $\Pi_{2}$ is a path on $Q$, and it can be verified that $\Pi_{2}$ is a $W_{v}$ path or both $u$ and $v$ appear in $\Pi_{2}$. In the latter case, a $W_{v}$ path from $x$ to $y$ on $Q$ is formed by simply omitting from $\Pi_{2}$ all vertices which appear between $u$ and $v$.

For the remaining case, we may assume without loss of generality that $y=v, x \neq u$, and the splitting is of type 2 or type 3 . Let $\Pi_{1}$ be a $W_{v}$ path from $x$ to $b$ on $P$, and let $\Pi_{2}$ be formed as above. If $u$ appears in $\Pi_{2}$, a $W_{v}$ path $\Pi_{3}$ from $x$ to $y$ on $P$ is obtained from $\Pi_{2}$ by inserting $v(=y)$ after $u$ and discarding all vertices of $\Pi_{2}$ which appear after $u$. If $u$ does not appear in $\Pi_{2}$ but $v$ does appear, $\Pi_{3}$ is obtained from $\Pi_{2}$ by discarding all vertices of $\Pi_{2}$ which appear after $v$. If neither $u$ nor $v$ appears in $\Pi_{2}, \Pi_{3}$ is obtained from $\Pi_{2}$ by inserting $v$ after $b$. In each case, $\Pi_{3}$ is a $W_{v}$ path from $x$ to $y$ on $Q$, and this completes the proof.

Finally, we show under very restrictive hypotheses that shortest paths are $W_{v}$ paths or $W_{e}$ paths. Here (in contrast to 2.3) shortness is not a metric notion but rather involves the combinatorial notion of length employed in $\S 1$.

Proposition 2.6. Suppose that $p$ and $q$ are vertices of a polyhedral cell-complex $\boldsymbol{K}$, and that $\Pi$ is a shortest path from $p$ to $q$ in $\boldsymbol{K}$ (that is, a path involving the smallest possible number of vertices).

If every cell of $\boldsymbol{K}$ is of diameter $\leqq 1$, then $I$ is a $W_{v}$ path.

If every cell of $\boldsymbol{K}$ is of diameter $\leqq 3$, then $\Pi$ is a $W_{e}$ path.

If $\boldsymbol{K}$ is the boundary complex of a simple d-polyhedron $P$ and every facet of $P$ is of diameter $\leqq 2$, then $\Pi$ is a $W_{v}$ path or $p$ and 
$q$ lie together in a facet $F$ of $P$. (In the latter case, $p$ and $q$ can be joined in $F$ by a path which is both a $W_{v}$ path and a shortest path in $K$. However, II itself need not be a $W_{v}$ path.)

Proof. Let $\Pi=\left(x_{0}, x_{1}, \cdots, x_{l}\right)$ and suppose that $\Pi$ is not a $W_{v}$ path. Then some cell $C$ of $\boldsymbol{K}$ includes $x_{i}$ and $x_{k}$ but neither $x_{i+1}$ nor $x_{k-1}$, where $0 \leqq i<i+1 \leqq k-1<k \leqq l$. If $\delta(C) \leqq 1^{14}$, then $\left[x_{i}, x_{k}\right] \in \boldsymbol{K}$ and $\left(x_{0}, \cdots, x_{i}, x_{k}, \cdots, x_{l}\right)$ is a path from $p$ to $q$ of length $<l$, contradicting the assumption that $I$ is a shortest path. This establishes the first assertion of 2.6 .

Under the hypotheses of the third assertion, we may assume that $C$ is a facet of $P$. Since $\Pi$ is a shortest path and $\delta(C) \leqq 2$, we see that $i+1=k-1$ and there is a vertex $y$ of $C$, not among the $x_{j}$ 's, such that $\left[x_{i}, y\right]$ and $\left[y, x_{k}\right]$ are both edges of $C$. Since $P$ is a simple $d$ polyhedron, each of $x_{i}$ and $x_{k}$ is incident to exactly $d$ edges of $P$ and to exactly $d-1$ edges of $C$, whence it follows that $i=0$ or $x_{i-1} \in C$ and also that $k=l$ or $x_{k+1} \in C$. If $x_{i-1} \in C$ or $x_{k+1} \in C$, we may use the fact that $\delta(C) \leqq 2$ to produce a path of length $<l$ from $p$ to $q$. Since this is impossible, it follows that $i=0, k=l$, and $\{p, q\} \subset C$. The statement in parentheses is then easily verified, and the proof of the third assertion is complete.

For the second assertion of 2.6 , let us assume that $\Pi$ is not a $W_{e}$ path, whence some cell $C$ of $\boldsymbol{K}$ contains the edges $\sigma_{i}\left(=\left[x_{i-1}, x_{i}\right]\right)$ and $\sigma_{k}$ of $\boldsymbol{K}$ but neither $\sigma_{i+1}$ nor $\sigma_{k-1}$, where $1 \leqq i<i+1 \leqq k-1<k \leqq l$. In fact, $i+1<k-1$, for otherwise $\sigma_{i+1} \subset C$. Thus we have $x_{i-1}, x_{k} \in C$ with $k \geqq i+3$, and since $\delta(C) \leqq 3$ it is possible to connect $p$ and $q$ by a path shorter than $I I$. The contradiction completes the proof.

Figure 2 depicts a complex in which every cell is of diameter $\leqq 2$, and yet no shortest path from $p$ to $q$ is a $W_{v}$ path. Figure 3

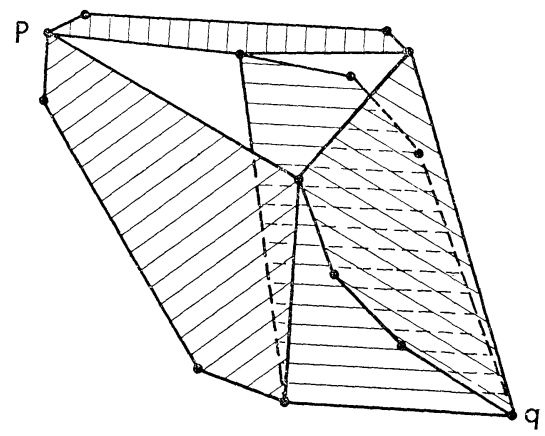

Figure 2

${ }^{14} \delta(C)$ is the diameter of $C$. 


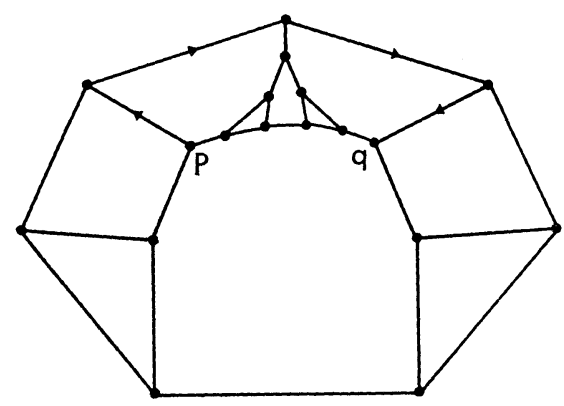

Figure 3

is the Schlegel diagram of a simple 3-polytope in which no shortest path from $p$ to $q$ is a $W_{v}$ path. $^{15}$

\section{REFERENCES}

1. P. Alexandroff and H. Hopf, Topologie I, Springer, Berlin, 1935.

2. T.A. Brown, Simple paths on convex polyhedra, Pacific J. Math. 11 (1961), 12111214.

3. M. Brückner, Über die Ableitung der allgemeinen Polytope und die nach Isomorphismus verschiedenen Typen der allgemeinen Achtzelle (Oktatope), Verhandelingen der Koninklijke Akademie van Wetenschappen te Amsterdam (Eerste Sectie), Deel X, No. 1 (1909), 29 pages +2 plates.

4. C. Carathéodory, Über den Variabilitätsbereich der Fourierschen Konstanten von positiven harmonischen Funktionen, Rend. Circ. Mat. Palermo, 32 (1911), 193-217.

5. G. B Dantzig, Linear programming and extensions, Princeton Univ. Press, Princeton, N. J., 1963.

6. D. Gale, Neighborly and cyclic polytopes, Proc. Symp. Pure. Math., 7, Convexity, Amer. Math. Soc., Providence, R. I., (1963), 225-232.

7. - On the number of faces of a convex polytope, Canad. J. Math. 16 (1964), 12-17.

8. B. Grünbaum, Convex polytopes, Wiley, N. Y., 1966.

9. B. Grünbaum and T.S. Motzkin, Longest simple paths in polyhedral graphs, J. London Math. Soc. 37 (1962), 152-160.

10. V. Klee, Diameters of polyhedral graphs, Canad. J. Math. 16 (1964), 602-614.

11. On the number of vertices of a convex polytope, Canad. J. Math. 16 (1964), 701-720.

12. - Heights of convex polytope, J. Math. Anal. Appl., 11 (1965), 176-190.

13. Convex polytopes and linear programming, Proceedings of the IBM Scientific Computing Symposium on Combinatorial Problems (March 16-18, 1964), 1966.

14. - Paths on polyhedra. I, J. Soc. Appl. Ind. Math. 13 (1965), 946-956.

15. L. A. Lyusternik, Convex figures and polyhedra, Dover, New York, 1963.

16. J.W. Moon and L. Moser, Simple paths on polyhedra, Pacific J. Math. 13 (1963), 629-631.

17. T.S. Motzkin, Comonotone curves and polyhedra, Abstract 111, Bull. Amer. Math. Soc. 63 (1957), 35.

${ }^{15}$ Here, of course, "shortest path" is in the sense of 2.6. D. Barnette [24] has produced a simple 3-polytope having only 14 vertices and at the same time having two vertices $p$ and $q$ such that no shortest path from $p$ to $q$ is a $W_{v}$ path. He has proved that 14 is the smallest number for which this is possible. 
18. E. Steinitz, Polyeder und Raumeinteilungen, Enzyklopädie der Mathematischen Wissenschaften, 3AB 12 (1922), 1-139.

19. E. Steinitz and H. Rademacher, Vorlesungen ïber die Theorie der Polyeder, Springer, Berlin, 1934.

20. W. T. Tutte, On Hamiltonian circuits, J. London Math. Soc. 21 (1946), 98-101.

21. - - A theorem on planar graphs, Trans. Amer. Math. Soc., 82 (1956) 99-116. 22. - A non-Hamiltonian planar graph, Acta Math. Acad. Sci. Hungar. 11 (1960), 371-375.

23. H. Whitney, A theorem on graphs, Ann. of Math. 32 (1931), 378-390.

24. D. Barnette, $W_{v}$ paiths on 3-polytopes, Journal of Combinatorial Theory, to appear. 25. V. Klee and D. Walkup, The d-step conjecture for polytopes of dimension $d<6$, to appear.

\section{UNIVERSITY OF WASHING'TON}

and

Boeing Scientific Research Laboratories

\section{Footnotes added in Proof.}

${ }^{16}$ In [25] there is constructed an unbounded 4-polyhedron which has 8 facets and is of diameter 5; necessarily (in view of 2.1) it has two vertices which cannot be joined by any $W_{v}$ path. Also in [25] is a proof that the following two statements are equivalent, though not necessarily on a dimension-for-dimension basis: any two vertices of a simple polytope can be joined by a $W_{v}$ path; on a $d$-polytope with $n$ facets, any two vertices can be joined by a path of length $\leqq n-d$.

17 D. Barnette [24] has proved that any two vertices of a 3-poiytope can be joined by two independent $W_{v}$ paths if they do not share an edge and by three independent $W_{v}$ path if they do not share a facet. D. Walkup has remarked that 2.4 can be used to prove that any two vertices $x$ and $y$ of a 3-polytope $P$ can be joined by a $W_{v}$ path on $P$. Choose a vertex $u$ different from $x$ and $y$ and a plane $H$ such that $H \cap P=\{u\}$. Then apply 2.4 to the unbounded polyhedron which is the image of $P$ under a projective transformation carrying $H$ onto the plane at infinity. 


\section{PACIFIC JOURNAL OF MATHEMATICS}

\section{EDITORS}

\author{
H. SAMELSON \\ Stanford University \\ Stanford, California \\ R. M. BLUMENTHAL \\ University of Washington \\ Seattle, Washington 98105
}

\author{
*J. DUGUNDJI \\ University of Southern California \\ Los Angeles, California 90007
}

RICHARD ARENS

University of California Los Angeles, California 90024

\section{ASSOCIATE EDITORS}
E. F. BECKENBACH
B. H. NEUMANN
F. WOLF
K. YoSIDA

\section{SUPPORTING INSTITUTIONS}

\author{
UNIVERSITY OF BRITISH COLUMBIA \\ CALIFORNIA INSTITUTE OF TECHNOLOGY \\ UNIVERSITY OF CALIFORNIA \\ MONTANA STATE UNIVERSITY \\ UNIVERSITY OF NEVADA \\ NEW MEXICO STATE UNIVERSITY \\ OREGON STATE UNIVERSITY \\ UNIVERSITY OF OREGON \\ OSAKA UNIVERSITY \\ UNIVERSITY OF SOUTHERN CALIFORNIA
}

\author{
STANFORD UNIVERSITY \\ UNIVERSITY OF TOKYO \\ UNIVERSITY OF UTAH \\ WASHINGTON STATE UNIVERSITY \\ UNIVERSITY OF WASHINGTON \\ * * * * \\ AMERICAN MATHEMATICAL SOCIETY \\ CHEVRON RESEARCH CORPORATION \\ TRW SYSTEMS \\ NAVAL ORDNANCE TEST STATION
}

Mathematical papers intended for publication in the Pacific Journal of Mathematics should be typewritten (double spaced). The first paragraph or two must be capable of being used separately as a synopsis of the entire paper. It should not contain references to the bibliography. Manuscripts may be sent to any one of the four editors. All other communications to the editors should be addressed to the managing editor, Richard Arens at the University of California, Los Angeles, California 90024.

50 reprints per author of each article are furnished free of charge; additional copies may be obtained at cost in multiples of 50 .

The Pacific Journal of Mathematics is published monthly. Effective with Volume 16 the price per volume (3 numbers) is $\$ 8.00$; single issues, $\$ 3.00$. Special price for current issues to individual faculty members of supporting institutions and to individual members of the American Mathematical Society: $\$ 4.00$ per volume; single issues $\$ 1.50$. Back numbers are available.

Subscriptions, orders for back numbers, and changes of address should be sent to Pacific Journal of Mathematics, 103 Highland Boulevard, Berkeley 8, California.

Printed at Kokusai Bunken Insatsusha (International Academic Printing Co., Ltd.), No. 6, 2-chome, Fujimi-cho, Chiyoda-ku, Tokyo, Japan.

\section{PUBLISHED BY PACIFIC JOURNAL OF MATHEMATICS, A NON-PROFIT CORPORATION}

The Supporting Institutions listed above contribute to the cost of publication of this Journal, but they are not owners or publishers and have no responsibility for its content or policies.

* Paul A. White, Acting Editor until J. Dugundji returns. 


\section{Pacific Journal of Mathematics}

\section{Vol. 17, No. 2 \\ February, 1966}

Henry A. Antosiewicz, Boundary value problems for nonlinear ordinary

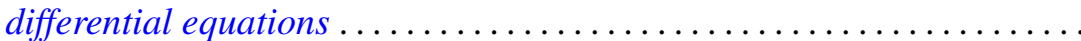

Bernard Werner Levinger and Richard Steven Varga, Minimal Gerschgorin sets. II ........................................

Paul Camion and Alan Jerome Hoffman, On the nonsingularity of complex matrices........................................ 211

J. Chidambaraswamy, Divisibility properties of certain factorials ........ 215

J. Chidambaraswamy, A problem complementary to a problem of Erdös .... 227

John Dauns, Chains of modules with completely reducible quotients ...... 235

Wallace E. Johnson, Existence of half-trajectories in prescribed regions and asymptotic orbital stability ............................ 243

Victor Klee, Paths on polyhedra. II . ........................ 249

Edwin Haena Mookini, Sufficient conditions for an optimal control problem in the calculus of variations ............................ 263

Zane Clinton Motteler, Existence theorems for certain quasi-linear elliptic equations........................................... 279

David Lewis Outcalt, Simple n-associative rings ............... 301

David Joseph Rodabaugh, Some new results on simple algebras ......... 311

Oscar S. Rothaus, Asymptotic properties of groups generation ........... 319

Ernest Edward Shult, Nilpotence of the commutator subgroup in groups admitting fixed point free operator groups .............

William Hall Sills, On absolutely continuous functions and the

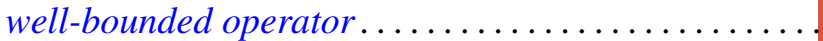

Joseph Gail Stampfli, Which weighted shifts are subnormal ..

Donald Reginald Traylor, Metrizability and completeness in normal Moore spaces 